\title{
La Formación Continuada en Drogodependencias
}

\author{
BecoñA, E.; LloPIS, J.J.; BOBES, J. \\ Comisión de Docencia de Socidrogalcohol
}

\section{RESUMEN}

La formación continuada ha sido una de las grandes inquietudes de los profesionales de las drogodependencias desde el primer momento. El autodidactismo y el reciclaje sin contrastar dominó la primera época para luego pasar a una formación reglada y de calidad. Las Sociedades Científicas primero y luego estas mismas coordinadamente con Universidad y administraciones autonómicas han conseguido que en estos momentos la formación de postgrado en España presente el mejor nivel de toda Europa, ya sea por medio de Masters Universitarios o mediante la formación de expertos en drogodependencias.

Socidrogalcohol como sociedad científica, con largo bagaje en la formación continuada y reciclaje de sus miembros, consciente del papel transcendental que debe desempeñar en la docencia ha decidido hacer una apuesta por la creación de un marco estable anual donde la formación de calidad y actualizada a las nuevas exigencias del mundo de las toxicomanías sean el objetivo fundamental, por lo que en este año 2000 inicia una nueva andadura con la Escuela de Otoño en Drogodependencias de Socidrogalcohol.

Palabras clave: formación continuada, España, Socidrogalcohol, profesionales, cursos.

\section{ABSTRACT:}

Continuous training has been one of the big worries of professionals in drug dependency since the very early stages times. Self-education and recycling without qualifications dominated the early stages before a regulated and excellent training became the norm. First it was the Scientific Associations themselves and then, working in conjunction with Universities and Autonomous Governments, they have ensured that at this present time postgraduate training in Spain presents the highest level in all of Europe either through university master=s degrees or through the training of experts in drugdependency.

Socidrogalcohol as a scientific association with a hefty baggage in continuous training and refreshment courses for its members, and well aware of the importance of the role which it must play in education has decided to support the creation of a stable annual framework where excellent training, in line with the actual demands of the world of drug addictions are the fundamental objectives and, therefore, now in 2000, it is initiating a new stage with the Socidrogalcohol Autumn School in Drugdependency.

Key words: Continuos training, Spain, Socidrogalcohol, training professionals, courses, workshops.
H oy podemos afirmar que en España los profesionales que trabajan el campo de las drogodependencias están en una etapa de madurez. Más de dos décadas de trabajo continuo en este campo por parte de un gran número de profesionales ha permitido consolidar conocimientos, técnicas, intervenciones, todo ello facilitado por disponer de recursos (no siempre suficientes) a través de la amplia red asistencial de drogodependencias de las distintas comunidades autónomas.

Pero esto no siempre ha sido así. A mediados de los 70 se produjo el viraje ineludible de los profesionales dedicados al tratamiento del alcoholismo hacia un nuevo tipo de dependencia con necesidades diferen- tes. La adicción a la heroína provoca tener que adaptar las técnicas de tratamiento y la necesidad de una formación que en esos momentos no se veía correspondida por la oferta oficial. Son las sociedades científicas y autonomías como Cataluña y el País Vasco quienes comienzan a dar respuesta a esa necesidad de formación propiciando encuentros profesionales y cursos de doctorado.

Ya plenamente contrastada la importancia que las drogodependencias adquieren como problema de salud y su extensión cuasi epidémica para determinados sectores de población la Oficina Regional Europea de la OMS, en 1985, valora la necesidad de formación adecuada para los profesionales de la salud y distribu- 
ye, entre sus países miembros, una encuesta sobre formación en drogodependencias, a través de la que se constata que en la mayoría de los países europeos los esfuerzos dedicados a la formación eran escasos y poco coordinados. Posteriormente recomienda que la formación en drogodependencias sea una parte esencial dentro de la formación sanitaria y la organización de cursos de postgrado para la formación especializada dentro de un marco multiprofesional.

Tras un período de formación basada fundamentalmente en la experiencia clínica, muchas veces con características de ensayo-error y sin grandes posibilidades de contraste ni reflexión, a finales de los 80 aparecen las primeras propuestas regladas de formación de postgrado en drogodependencias. En Madrid la Complutense, La Universidad de Barcelona y más tarde Deusto en Bilbao, ofertan los primeros Masters en Drogodependencias como respuesta universitaria a la necesidad de los profesionales.

La formación en drogodependencias ha sido un tema de gran relevancia en la década pasada y se han dedicado muchos esfuerzos y medios. Esta formación se ha realizado fundamentalmente a través de dos vías. La primera ha sido mediante la enseñanza de postgrado, en forma de Master o Cursos de Especialización en Drogodependencias, de un año o dos de duración. La mayoría de ellos se han visto favorecidos por una financiación parcial o casi completa por parte de los distintos Planes Autonómicos en Drogodependencias.

El interés de la administración era claro: formar del mejor modo posible a los profesionales que trabajaban en su red asistencial y formar de modo especializado a otros profesionales que en los años siguientes iban a trabajar en este campo. Gracias a ello han surgido un gran número de cursos de postgrado a lo largo de la geografía nacional, como todos conocemos.

La situación ha cambiado en los últimos años una vez que estaban formados la mayor parte de los profesionales en un corpus de conocimientos teórico-prácticos básicos. En lo anterior la colaboración entre las distintas universidades y los planes autonómicos ha sido muy fructífera y ha permitido dar un salto cualitativo en la formación de postgrado, a diferencia de lo que ha ocurrido en los países de nuestro entorno.

También, y como complemento a la formación anterior, las sociedades científicas, y especialmente Socidrogalcohol, han llevado a cabo una labor continua de formación de sus miembros, todos relacionados con el campo de las drogodependencias o las adicciones. Sus Congresos anuales han servido de lugar de encuentro de la mayoría de los profesionales que trabajan en drogodependencias en España y de complemento a la formación de sus miembros. Nótese que se va ya por su 27 edición, lo que implica una fuerte consolidación. En sus congresos se han ido presen- tando los últimos avances en este campo y la participación de sus socios ha sido muy amplia.

En los últimos años se aprecia un ligero cambio al panorama anterior. Por una parte, la desaparición de varios de los Masters y cursos de especialización que se pusieron en marcha en los años previos. Desde la Universidad esa desaparición en unos casos ha conllevado una real desaparición y en otros una transformación en Institutos de Drogodependencias, en este caso más orientados a la investigación y a la formación continuada. Paralelamente, las sociedades científicas han incrementado su formación en forma de cursos monográficos, simposium y, en el caso específico de Socidrogalcohol, un gran incremento de las actividades de las secciones autonómicas en forma de Jornadas Autonómicas. Esto es una clara prueba de la necesidad de continuar y profundizar en la formación continuada.

En todo lo anterior ha habido siempre un interés mutuo entre los profesionales, las sociedades científicas, las universidades y los responsables autonómicos o del propio Plan Nacional sobre Drogas. Este ha elaborado varios documentos analizando esta cuestión en los últimos años. En 1993 la Comisión interautonómica del PND sobre formación, en sus conclusiones, recomienda a los Programas de Especialidades del Área de Salud crear la Especialización en Drogodependencias y el reciclaje de los profesionales en activo, señalando a los propios profesionales de los servicios de toxicomanías como los más cualificados para impartir la docencia coordinadamente con la Universidad.

También la Unión Europea, y específicamente el Observatorio Europeo de Drogas se han ocupado de este tema elaborando varios documentos. En ellos se observa la necesidad de profundizar cada vez más en la formación continuada por una parte y, por otra, en hacer dicha formación flexible y adaptada a las necesidades de cada momento, especialmente para los profesionales que trabajan en un campo como el nuestro tan cambiante.

Las razones anteriores, que van en la línea de la evolución que venimos teniendo en los últimos años en el campo de la formación, así como la demanda creciente de cursos de formación especializados, el surgimiento de nuevas sustancias, nuevos tratamientos y abordajes, así como nuestra obligación de tener una formación continuada, justifican la puesta en marcha por parte de Socidrogalcohol de la Escuela de Otoño Socidrogalcohol.

Como Sociedad Científica Española de Estudios sobre el Alcohol, el Alcoholismo y las otras Toxicomanías, siendo una de sus funciones principales la formación continuada de sus miembros y con el convencimiento pleno del gran papel que le corresponde a las sociedades científicas de prestigio en el 
reciclaje de los profesionales, hacemos desde Socidrogalcohol una apuesta por la formación continuada de forma estable dándole una entidad que pueda servir como referente y punto de encuentro anual de las nuevas generaciones de profesionales en drogodependencias creando la Escuela de Otoño Socidrogalcohol en Drogodependencias.

La escuela de otoño Socidrogalcohol nace con los siguientes objetivos:

Crear un marco estable donde llevar a cabo talleres de formación en los aspectos más emergentes en relación al cambiante mundo de las drogodependencias.

Facilitar la formación continuada mediante talleres monográficos simultáneos de profundización teórico práctica.

Dar continuidad a los distintos seminarios y cursos de formación que hasta el momento se han venido realizando de forma intermitente.

Desarrollar una formación continuada de calidad, evaluada y acreditada tanto por Socidrogalcohol como por los estamentos universitarios pertinentes.

Socidrogalcohol apuesta por la continuidad de aquellas actividades de formación que han tenido una gran acogida y una evaluación positiva de sus participantes de forma que aquellos cursos bandera de la sociedad se integran en la Escuela de Otoño propiciando la planificación de objetivos con continuidad y la programación de distintos niveles de formación en un mismo aspecto. Los seminarios de Entrevista Motivacional, los cursos de Formación en Psicoterapia Grupal del Alcoholismo, La intervención precoz desde Atención primaria o los cursos de formación en psicoestimulantes, que tan buena acogida han tenido en anteriores ediciones pasan a ser, entre otros de nueva oferta, el estandarte de la Escuela de Otoño de Socidrogalcohol.

El formato de la Escuela de Otoño de Socidrogalcohol posibilita una formación más personalizada, por el numero limitado de participantes, facilitando la necesaria flexibilidad de objetivos y contenidos a fin de acomodarlos al cambiante fenómeno de las adicciones. Orientada a los profesionales con una sólida formación y a aquellos que quieran entrenarse en técnicas novedosas para su práctica clínica, su práctica preventiva o su práctica de reinserción social. Y, también, orientado a los distintos profesionales que trabajan en el campo de las drogodependencias (médicos, psicólogos, trabajadores sociales, enfermeras, educadores, etc.).

Formación basada en la participación, flexibilidad, actividad y realidad, sobre la base de seminarios de trabajo para grupos reducidos con necesidades formativas en la actividad diaria. Enriqueciendo la aportación universitaria con la participación de profesionales de reconocido prestigio que intervienen desde la asistencia y la prevención para aportar la dimensión práctica del contacto permanente con las drogodependencias.

El entorno en el que se va a desarrollar la Escuela de Otoño propicia la combinación de la formación con una estancia lúdica y cultural en una zona de la costa mediterránea con el atractivo necesario para convertirse en el lugar de encuentro de profesionales que deseamos.

De esta forma se continua con la línea que se ha seguido en los últimos años en la formación, la escuela de otoño Socidrogalcohol colaboraría con las instituciones relacionadas con el campo de las drogodependencias (Plan Nacional sobre Drogas, Planes de Drogas autonómicos) y con las Universidades del entorno en donde se ubica la escuela. Esto facilita el reconocimiento de los créditos académicos por una institución universitaria, al mismo tiempo que recibe los créditos de Socidrogalcohol. Con todo ello se consigue poder seguir manteniendo esa tan importante unión entre el mundo profesional, el académico y el institucional en beneficio de la formación de los profesionales para una atención a las drogodependencias eficaz y eficiente. 
